\title{
RAYLEIGH'S QUOTIENT FOR MULTIPLE CRACKED BEAM AND APPLICATION
}

\author{
Nguyen Tien Khiem, Tran Thanh Hai \\ Institute of Mechanics, VAST
}

\begin{abstract}
Rayleigh's quotient for Euler-Bernoulli multiple cracked beam with different boundary conditions has been derived from the governed equation of free vibration. An appropriate choosing of approximate shape function in terms of mode shape of uncracked beam and specific functions satisfying conditions at cracks and boundaries leads to an explicit expression of natural frequencies through crack parameters that can simplify not only the analysis of natural frequencies of cracked beam but also the crack detection problem. Numerical analysis of natural frequencies of the cracked beam by using the obtained expression in comparison with the well known methods such as the characteristic equation and finite element method shows their good agreement. The analytical expression of natural frequencies applied to the crack detection problem allows the result of detection to be improved.
\end{abstract}

Key words: Natural frequencies, multiple cracked beam, Rayleigh's quotient.

\section{INTRODUCTION}

Recently, a significant effort of researchers as well as engineers has been devoted to solve forward and inverse problems in vibration of cracked beam. The most important tool for solving the problems is so-called the characteristic equation relating implicitly natural frequencies with crack parameters. It has been shown in several studies, for instance $[1,2]$ that for a beam with single crack of extent $\gamma$ at position $x_{c}$ the characteristic equation has the form

$$
F_{0}(\omega)+\gamma F_{1}\left(\omega, x_{c}\right)=0
$$

where $F_{0}$ and $F_{1}$ are functions given by boundary conditions of the beam. If the crack extent $\gamma$ is small, from the equation an explicit expression of natural frequencies through crack parameters can be derived as

$$
\Delta \omega_{k}=\omega_{k}-\omega_{k 0}=\gamma g\left(\omega_{k 0}, x_{c}\right)
$$

with $\omega_{k}, \omega_{k 0}$ are frequencies of cracked and uncracked beam respectively. This is an approximation available only for small extent of single crack $[3,4]$. J. Fernandez-Saez, L Rubio and C. Navarro [5] have obtained a formulae for fundamental frequency of simply supported beam using Rayleigh's method without assumption on smallness of crack depth. Comparison of the obtained analytical result [5] with numerical one shows that both the results are almost identical for the crack depth reached $75 \%$ beam thickness. 
The characteristic equation (1) has been developed for multiple cracked Bernoulli-Euler beam in $[6,7]$ by using the transfer matrix method and obtained explicitly in an analytical form in [8]. The author of reference [9] has developed the finite element model of multiple cracked beam and established the characteristic equation accordingly to the FEM. The approximate equations similar to the equation (2) for multiple cracked beam was also established in $[2,7]$ assuming that the mode shapes of cracked beam remain the same as those of uncracked one. An important idea arisen from [5] is to obtain an explicit expression of natural frequencies of higher modes with other boundary conditions and for multiple cracked beam based on the choosing mode shapes of cracked beam. This idea has been developed in [10] to obtain higher frequencies (but not in analytical form) for no uniform beam using Galerkin's method. This paper is devoted to derive an analytical expression for any natural frequency of multiple cracked beam with arbitrary boundary conditions using Rayleigh method. Theoretical section is devoted to establish general form of Rayleigh's quotient for multiple cracked beam, that is applied in subsequent section to obtain analytical expressions of natural frequencies of the cracked beam through crack parameters for different cases of the classical boundary conditions. Numerical investigation is presented in the last section for comparison in order to validate the theory.

\section{THEORY}

Consider an uniform with the material and geometrical constants: Young's modulus $E$, mass density $\rho$, length $L$, cross section area and moment of inertia $F, I$ and with given arbitrarily boundary conditions at the ends $x=0$ and $x=L$.

It is well known that for such the beam, the eigenparameters consisting of natural frequencies and corresponding mode shapes $\left\{\omega_{k}^{2}, \phi_{k}(x), k=1,2, \ldots.\right\}$ satisfying the equation

$$
\phi_{k}^{(I V)}(x)-\lambda_{k}^{4} \phi_{k}(x)=0, \lambda_{k}^{4}=\frac{\rho F \omega_{k}^{2}}{E I}, k=1,2, \ldots
$$

Suppose, furthermore, that the beam has been cracked at the positions $\left(e_{1}, \ldots, e_{N}\right)$ and the cracks are modeled as rotational springs of stiffness $K_{1}, \ldots, K_{N}$ respectively. Dividing the beam into $\mathrm{N}$ segments $\left(x_{j-1}, x_{j}\right), j=1, \ldots, N$, each of those contains a crack $\left(e_{j}, K_{j}\right)$ so that $x_{j-1} \prec e_{j} \prec x_{j}, j=1, \ldots, N$ and $x_{0}=0, x_{N}=L$.

Considering equation (1) in $\mathrm{j}$-th beam segment $\left(x_{j-1}, x_{j}\right)$ and denoting the mode shape for this segment by $\phi_{k j}(x)$, one will have

$$
\phi_{k j}^{(I V)}(x)-\lambda_{k}^{4} \phi_{k j}(x)=0, x \in\left(x_{j-1}, x_{j}\right) .
$$

Multiplying both sides of the equations by $\phi_{k j}(x)$, then taking integration along the interval $\left(x_{j-1}, x_{j}\right)$ yields

$$
\omega_{k}^{2}=(E I / \rho F) \sum_{j=1}^{N} a_{k j} / \sum_{j=1}^{N} b_{k j}, k=1,2,3, \ldots
$$


where

$$
a_{k j}=\int_{x_{j-1}}^{x_{j}} \phi_{k j}^{(I V)}(x) \phi_{k j}(x) d x, b_{k j}=\int_{x_{j-1}}^{x_{j}} \phi_{k j}^{2}(x) d x .
$$

If functions $\phi_{k j}, \phi_{k j}^{\prime}, \phi_{k j}^{\prime \prime}, \phi_{k j}^{\prime \prime \prime}$ are continuous in the interval $(a, b)$, one has

$$
\int_{a}^{b} \phi_{k j}^{(I V)}(x) \phi_{k j}(x) d x=\int_{a}^{b} \phi_{k j}^{2}(x) d x+B_{k j}(b)-B_{k j}(a)
$$

where

$$
B_{k j}(x)=\phi_{k j}^{\prime \prime \prime}(x) \phi_{k j}(x)-\phi_{k j}^{\prime \prime}(x) \phi_{k j}^{\prime}(x) .
$$

Applying equations $(7),(8)$ to two segments $\left(x_{j-1}, \bar{x}_{j}\right),\left(\bar{x}_{j}, x_{j}\right)$ for arbitrary $\bar{x}_{j} \in$ $\left(x_{j-1}, x_{j}\right)$ leads the first integral in $(6)$ to

$$
a_{k j}=\int_{x_{j-1}}^{x_{j}} \phi_{k j}^{\prime \prime 2}(x) d x+\left[B_{k j}\left(\bar{x}_{j}^{-}\right)-B_{k j}\left(\bar{x}_{j}^{+}\right)\right]+\left[B_{k j}\left(x_{j}^{-}\right)-B_{k j}\left(x_{j-1}^{+}\right)\right]
$$

and, in consequence,

$$
\begin{aligned}
\sum_{j=1}^{N} a_{k j}= & \sum_{j=1}^{N} \int_{x_{j-1}}^{x_{j}} \phi_{k j}^{\prime \prime 2}(x) d x+\sum_{j=1}^{N-1}\left[B_{k j}\left(x_{j}\right)-B_{k, j+1}\left(x_{j}\right)\right]+ \\
& +\sum_{j=1}^{N}\left[B_{k j}\left(\bar{x}_{j}^{-}\right)-B_{k j}\left(\bar{x}_{j}^{+}\right)\right]+\left[B_{k N}\left(x_{N}\right)-B_{k 1}\left(x_{0}\right)\right] .
\end{aligned}
$$

Because the beam is uniform (continuous) at the joints $x_{j}, j=1, \ldots, N-1$, the functions $\phi_{k j}(x)$ satisfy conditions

$$
\phi_{k, j-1}\left(x_{j}^{-}\right)=\phi_{k j}\left(x_{j}^{+}\right) ; \phi_{k, j-1}^{\prime}\left(x_{j}^{-}\right)=\phi_{k j}^{\prime}\left(x_{j}^{+}\right) ; \phi_{k, j-1}^{\prime \prime}\left(x_{j}^{-}\right)=\phi_{k j}^{\prime \prime}\left(x_{j}^{+}\right) ; \phi_{k, j-1}^{\prime \prime \prime}\left(x_{j}^{-}\right)=\phi_{k j}^{\prime \prime \prime}\left(x_{j}^{+}\right)
$$

and following conditions at the crack position $e_{j}$ :

$$
\begin{gathered}
\phi_{k j}\left(e_{j}^{-}\right)=\phi_{k j}\left(e_{j}^{+}\right)=\phi_{k j}\left(e_{j}\right) ; E I \phi_{k j}^{\prime \prime \prime}\left(e_{j}^{-}\right)=E I \phi_{k j}^{\prime \prime \prime}\left(e_{j}^{+}\right)=E I \phi_{k j}^{\prime \prime \prime}\left(e_{j}\right) ; \\
K_{j}\left[\phi_{k j}^{\prime}\left(e_{j}^{+}\right)-\phi_{k j}^{\prime}\left(e_{j}^{-}\right)\right]=E I \phi_{k j}^{\prime \prime}\left(e_{j}^{+}\right)=E I \phi_{k j}^{\prime \prime}\left(e_{j}^{-}\right)=E I \phi_{k j}^{\prime \prime}\left(e_{j}\right) .
\end{gathered}
$$

It's not difficult to verify that the conditions (8) lead to $\sum_{j=1}^{N-1}\left[B_{k j}\left(x_{j}\right)-B_{k, j+1}\left(x_{j}\right)\right]=0$ and due to conditions (11) one has

$$
\left.\left.\sum_{j=1}^{N}\left[B_{k j}^{(} e_{j}^{-}\right)-B_{k j}^{(} e_{j}^{+}\right)\right]=\sum_{j=1}^{N}\left[\gamma_{j} \phi^{\prime \prime 2}\left(e_{j}\right)\right], \gamma_{j}=E I / K_{j} .
$$


Therefore, the equation (9) can be rewritten as

$$
\sum_{j=1}^{N} a_{k j}=\sum_{j=1}^{N}\left[\int_{x_{j-1}}^{x_{j}} \phi_{k j}^{\prime \prime 2}(x) d x+\gamma_{j} \phi_{k j}^{\prime \prime 2}\left(x_{j}\right)\right]+\left[B_{k N}(L)-B_{k 1}(0)\right],
$$

and the equation (5) gets the form

$$
\omega_{k}^{2}=\frac{E I}{\rho F} \frac{\sum_{j=1}^{N}\left[\int_{x_{j-1}}^{x_{j}} \phi_{k j}^{\prime \prime 2}(x) d x+\gamma_{j} \phi_{k j}^{\prime \prime 2}\left(x_{j}\right)\right]+\left[B_{k N}(L)-B_{k 1}(0)\right]}{\sum_{j=1}^{N}\left[\int_{x_{j-1}}^{x_{j}} \phi_{k j}^{2}(x) d x\right]} .
$$

This is Rayleigh's quotient generalized for multiple cracked uniform beam with arbitrary boundary conditions, some particular cases of which have been given in [10]. The equations (12), in fact, represent a relationship between unknown natural frequencies and corresponding mode shapes that allows to calculate natural frequencies by given mode shapes. However, as it is well known, finding an exact mode shape is strictly related to finding the natural frequency. An exact expression of the mode shape for multiple cracked beam has been obtained in [7], but it contains unknown natural frequency so that substituting the exact mode shape into equation (12) leads the latter into an identity. The major idea of the well known Rayleigh's method is to choose a trial shape function satisfying only boundary conditions instead of the unknown mode shape for calculating natural frequencies. Although such calculated natural frequencies are approximate, it may be closely approached to exact frequencies by a proper choosing the trial shape function. Furthermore, in the case of cracked beam, the calculated from Rayleigh's quotient natural frequencies give an explicit expression of crack parameters that is useful not only for analysis of natural frequencies but also to detect crack positions and depth. Below, a combination of mode shape for uncracked beam with specially chosen functions satisfying both the conditions (11) at cracks and arbitrary boundary conditions is developed to obtain an analytical expression of any natural frequencies through crack parameters.

Based on most importance for cracked beam that only its slope is discontinuous at the crack position, the mode shape functions are assumed to be chosen in the form

$$
\phi_{k j}(x)=\phi_{k 0}(x)+\phi_{k j}^{c}(x),
$$

where $\phi_{k 0}(x)$ are mode shapes of uncracked beam, continuous together with its derivatives $\phi_{k j 0}^{\prime}(x), \phi_{k j 0}^{\prime \prime}(x), \phi_{k j 0}^{\prime \prime \prime}(x)$ in whole the beam $(0, L)$ and linear functions

$$
\phi_{k j}^{c}(x)=C_{k j} x+D_{j k}+\left\{\begin{array}{c}
0, \quad x_{j-1} \leq x \prec e_{j} \\
\gamma_{j} \phi_{k 0}^{\prime \prime}\left(x_{j}\right) S\left(x-e_{j}\right), \quad e_{j} \prec x \leq x_{j}
\end{array}\right.
$$

with constants $C_{k j}, D_{k j}$ and function $S(x)=[\sinh \lambda x+\sin \lambda x] / 2 \lambda$, satisfying conditions: $S(0)=S^{\prime \prime}(0)=S^{\prime \prime \prime}(0)=0, S^{\prime}(0)=1$. Substituting equations (13), (14) into equations (10), (11) yields

$$
C_{k, j+1} x_{j}+D_{k, j+1}=C_{k j} x_{j}+D_{k j}+\gamma_{j} \phi_{k 0}^{\prime \prime}\left(x_{j}\right) S\left(x_{j}-e_{j}\right),
$$




$$
C_{k, j+1}=C_{k j}+\gamma_{j} \phi_{k j}^{\prime \prime}\left(e_{j}\right) S^{\prime}\left(x_{j}-e_{j}\right), j=1,2, \ldots, N,
$$

that in consequence lead to the expressions

$$
\begin{gathered}
C_{k j}=C_{k 1}+\sum_{i=1}^{j-1} \gamma_{i} \phi_{k 0}^{\prime \prime}\left(e_{i}\right) S^{\prime}\left(x_{i}-e_{i}\right), D_{k j}=D_{k 1}+\sum_{i=1}^{j-1} \gamma_{i} \phi_{k 0}^{\prime \prime}\left(e_{i}\right) \bar{S}\left(x_{i}-e_{i}\right), \\
\bar{S}\left(x_{i}-e_{i}\right)=\left[S\left(x_{i}-e_{i}\right)-x_{i} S^{\prime}\left(x_{i}-e_{i}\right)\right], \forall j=1, \ldots, N .
\end{gathered}
$$

with two arbitrary constants $C_{k 1}, D_{k 1}$ which would be determined from boundary conditions.

To calculate frequencies by equation (12) with the chosen mode shapes (13)-(15), at first, let's consider the last term in numerator of equation (12) that can be expressed as

$$
\left[B_{k N}\left(x_{N}\right)-B_{k 1}\left(x_{0}\right)\right]=\left[B_{k N}(L)-B_{k 1}(0)\right]=B_{k}^{0}+B_{k}^{c}-\sum_{j=1}^{N} \gamma_{j} \beta_{k j} \phi_{k 0}^{\prime 2}\left(e_{j}\right),
$$

where

$$
\begin{gathered}
B_{k}^{0}=\phi_{k 0}^{\prime \prime \prime}(L) \phi_{k 0}(L)-\phi_{k 0}^{\prime \prime}(L) \phi_{k 0}^{\prime}(L)-\phi_{k 0}^{\prime \prime \prime}(0) \phi_{k 0}(0)+\phi_{k 0}^{\prime \prime}(0) \phi_{k 0}^{\prime}(0), \\
B_{k}^{c}=\left[\phi_{k 0}^{\prime \prime \prime}(L) L-\phi_{k 0}^{\prime \prime}(L)+\phi_{k 0}^{\prime \prime}(0)\right] C_{k 0}+\left[\phi_{k 0}^{\prime \prime \prime}(L)-\phi_{k 0}^{\prime \prime \prime}(0)\right] D_{k 0}, \\
\beta_{k j}=\left[\phi_{k 0}^{\prime \prime \prime}(L)\left(e_{j}-L\right)+\phi_{k 0}^{\prime \prime}(L)\right] / \phi_{k 0}^{\prime \prime}\left(e_{j}\right) .
\end{gathered}
$$

The equations (17) show that $B_{k}^{0}$ is derived from given boundary conditions at the beam ends separately for uncracked beam, the determined by equations (18), (19) term $B_{k}^{c}$ and non-dimensional coefficient $\beta_{k j}$ express a combined effect of boundary conditions and cracks on natural frequencies. In some particular cases shown below the terms $B_{k}^{0}, B_{k}^{c}$, $\beta_{k j}$ can be annulled by choosing the constants $C_{k 0}, D_{k 0}$ accordingly to given boundary conditions of the beam. Thus, the complete numerator of of the equation (10) can be written in general form

$$
\sum_{j=1}^{N}\left[\int_{x_{j-1}}^{x_{j}} \phi_{k j}^{\prime \prime 2}(x) d x+\gamma_{j} \phi_{k j}^{\prime \prime 2}\left(x_{j}\right)\right]=\int_{0}^{L} \phi_{k 0}^{\prime \prime 2}(x) d x+\sum_{j=1}^{N} \gamma_{j}\left(1-\beta_{k j}\right) \phi_{k j}^{\prime \prime 2}\left(x_{j}\right)+B_{k}^{0}+B_{k}^{c}
$$

Now let's go to calculate the denominator of equation (10) using the mode shapes (14), (15). First, it can be expressed as

$$
\sum_{j=1}^{N}\left[\int_{x_{j-1}}^{x_{j}} \phi_{k j}^{2}(x) d x\right]=\sum_{j=1}^{N}\left[\int_{x_{j-1}}^{x_{j}} \hat{\phi}_{k 0}^{2}(x) d x+\Psi_{k j}\right]=\int_{0}^{L} \hat{\phi}_{k 0}^{2}(x) d x+\Psi_{1}+\Psi_{2},
$$

where

$$
\begin{gathered}
\Psi_{1}=\frac{2 E I}{\rho F \omega_{k 0}^{2}}\left[B_{k}^{c}+\sum_{j=1}^{N} \gamma_{j}\left(1-\beta_{k j}\right) \phi_{k 0}^{\prime \prime 2}\left(e_{j}\right)\right] \\
\Psi_{2}=A_{k}^{c}+\sum_{j=1}^{N} \gamma_{j}^{2} f_{k j} \phi_{k 0}^{\prime \prime 2}\left(e_{j}\right)+\sum_{j=2}^{N} \sum_{i=1}^{j-1} \gamma_{j} \gamma_{i} h_{j i} \phi_{k 0}^{\prime \prime}\left(e_{i}\right) \phi_{k 0}^{\prime \prime}\left(e_{j}\right)
\end{gathered}
$$


with $B_{k}^{c}, \beta_{k j}$ given by (18), (19) and

$$
\begin{gathered}
A_{k}^{c}=C_{k 0}^{2} L^{3} / 3+C_{k 0} D_{k 0} L^{2}+D_{k 0}^{2} L, \\
h_{j i}=\left(L-e_{j}\right)\left[\left(L-e_{i}\right)^{2}+\left(L-e_{i}\right)\left(L-e_{j}\right)-\left(e_{j}-e_{i}\right)^{2}\right] / 3, \\
f_{k j}=\frac{\left(L-e_{j}\right)^{3}}{3}\left[1+\frac{\left(2 L-e_{j}\right) C_{k 0}+3 D_{k 0}}{\left(L-e_{j}\right) \gamma_{j} \phi_{k 0}^{\prime \prime}\left(e_{j}\right)}\right] .
\end{gathered}
$$

Finally, generalized Rayleigh's quotient (10) for multiple cracked beam with mode shapes chosen in the form (14), (15) can be written in the form

$$
\omega_{k}^{2}=\frac{E I}{\rho F} \frac{\int_{0}^{L} \phi_{k 0}^{\prime \prime 2}(x) d x+\sum_{j=1}^{N}\left[\gamma_{j}\left(1-\beta_{k j}\right) \phi_{k 0}^{\prime 2}\left(e_{j}\right)\right]+B_{k}^{0}+B_{k}^{c}}{\int_{0}^{L} \phi_{k 0}^{2}(x) d x+\Psi_{1}\left[\gamma_{j} \phi_{k 0}^{2}\left(e_{j}\right)\right]+\Psi_{2}\left[\gamma_{j} \phi_{k 0}^{\prime \prime 2}\left(e_{j}\right)\right]},
$$

that is an analytical expression of natural frequency of cracked beam through crack parameters for arbitrary boundary conditions and mode shape of uncracked beam. Note that $\Psi_{1}, \Psi_{2}$ are linear and bilinear forms of crack extent vector $\left(\gamma_{1}, \ldots, \gamma_{N}\right)$ with coefficients being functions of crack positions $\left(e_{1}, \ldots, e_{N}\right)$. For uncracked beam, equation (26) leads to the well known Rayleigh's quotient

$$
\omega_{k 0}^{2}=\frac{E I}{\rho F}\left[\int_{0}^{L} \phi_{k 0}^{\prime \prime 2}(x) d x+B_{k}^{0}\right] / \int_{0}^{L} \phi_{k 0}^{2}(x) d x .
$$

At the end of this theoretical section, it has to emphasize that all equations obtained above are free from any assumption on the boundary conditions at the beam ends. Therefore, it provides a tool for investigation of either cracked or intact beam with different cases of boundary supports. In the next section, some cases of the classical boundary conditions are considered in more details.

\section{APPLICATION}

Before application of the developed theory to some particular cases, let's consider the classical boundary conditions applied for mode shape chosen in the form of (15) rewritten as

with

$$
\phi_{k}(x)=\phi_{k 0}(x)+\hat{\phi}_{k}(x)
$$

$$
\begin{gathered}
\hat{\phi}_{k}(x)=\hat{\phi}_{k j}(x), \text { for } x \in\left[x_{j-1}, x_{j}\right], j=1, \ldots, N ; \\
\hat{\phi}_{k j}(x)=C_{k j} x+D_{k j}+ \begin{cases}\gamma_{j}\left(e_{j}-x\right) \phi_{k 0}^{\prime \prime}\left(e_{j}\right) & \text { for } x \in\left[x_{j-1}, e_{j}\right) ; \\
0 & \text { for } x \in\left(e_{j}, x_{j}\right],\end{cases}
\end{gathered}
$$

that result in $\phi_{k}^{\prime \prime}(x)=\phi_{k 0}^{\prime \prime}(x) ; \phi_{k}^{\prime \prime \prime}(x)=\phi_{k 0}^{\prime \prime \prime}(x), \forall x \in[0, L]$ and

$$
\phi_{k}(0)=\phi_{k 0}(0)+D_{k 0} ; \phi_{k}^{\prime}(0)=\phi_{k 0}(0)+C_{k 0} ; \phi_{k}^{\prime}(L)=\phi_{k 0}^{\prime}(L)+C_{k 0}+\sum_{j=1}^{N} \gamma_{j} \phi_{k 0}^{\prime \prime}\left(e_{j}\right) ;
$$




$$
\phi_{k}(L)=\phi_{k 0}(L)+C_{k 0} L+D_{k 0}+\sum_{j=1}^{N} \gamma_{j}\left(L-e_{j}\right) \phi_{k 0}^{\prime \prime}\left(e_{j}\right) .
$$

The following classical boundary conditions for a beam are well known:

- Simple supports: $\phi_{k}(0)=\phi_{k}^{\prime \prime}(0)=\phi_{k}(L)=\phi_{k}^{\prime \prime}(L)=0$;

- Cantilever: $\phi_{k}(0)=\phi_{k}^{\prime}(0)=\phi_{k}^{\prime \prime \prime}(L)=\phi_{k}^{\prime \prime}(L)=0$;

- Free ends: $\phi_{k}^{\prime \prime \prime}(0)=\phi_{k}^{\prime \prime}(0)=\phi_{k}^{\prime \prime \prime}(L)=\phi_{k}^{\prime \prime}(L)=0$;

- Clamped ends: $\phi_{k}(0)=\phi_{k}^{\prime}(0)=\phi_{k}(L)=\phi_{k}^{\prime}(L)=0$.

If the mode shape of uncracked beam $\phi_{k 0}(x)$ satisfying the given above classical boundary conditions has been used, one has two constants $C_{k 0}, D_{k 0}$ to be chosen from the conditions

- Simple supports:

$$
\phi_{k}(0)=D_{k 0}=0, \phi_{k}(L)=C_{k 0} L+D_{k 0}+\sum_{j=1}^{N} \gamma_{j}\left(L-e_{j}\right) \phi_{k 0}^{\prime \prime}\left(e_{j}\right)=0
$$

- Cantilever:

$$
\phi_{k}(0)=D_{k 0}=0, \phi_{k}^{\prime}(0)=C_{k 0}=0
$$

- Free ends:

$$
\phi_{k}^{\prime \prime \prime}(0)=\phi_{k 0}^{\prime \prime \prime}(0)=0 ; \phi_{k}^{\prime \prime}(0)=\phi_{k 0}^{\prime \prime}(0)=0 ; \phi_{k}^{\prime \prime \prime}(L)=\phi_{k 0}^{\prime \prime \prime}(L)=0 ; \phi_{k}^{\prime \prime}(L)=\phi_{k 0}^{\prime \prime}(L)=0 .
$$

- Clamped ends:

$$
\begin{gathered}
\phi_{k}(0)=D_{k 0}=0, \phi_{k}(L)=C_{k 0} L+D_{k 0}+\sum_{j=1}^{N} \gamma_{j}\left(L-e_{j}\right) \phi_{k 0}^{\prime \prime}\left(e_{j}\right)=0 ; \\
\phi_{k}^{\prime}(0)=C_{k 0}=0 ; \phi_{k}^{\prime}(L)=C_{k 0}+\sum_{j=1}^{N} \gamma_{j} \phi_{k 0}^{\prime \prime}\left(e_{j}\right)=0 .
\end{gathered}
$$

From the latter equations it can be seen that in the case of simple supports and cantilever the constants $C_{k 0}, D_{k 0}$ will be chosen uniquely. For the free-free ends beam, the constants $C_{k 0}, D_{k 0}$ may be chosen arbitrary. For clamped end beam, the choosing of two constants is inadequate to satisfy four conditions $\phi_{k}(0)=\phi_{k}^{\prime}(0)=\phi_{k}(L)=\phi_{k}^{\prime}(L)=0$. Because of this uncertainty that requires a separated study, only the simply supported and cantilevered beams are considered in this work.

\subsection{Simply supported beam}

For simple supports at the beam ends, the boundary conditions would be satisfied if it is chosen $\phi_{k 0}(x)=\sin (k \pi x / L), k=1,2, \ldots$ and

$$
D_{k 0}=0, C_{k 0}=\sum_{j=1}^{N}\left(\frac{e_{j}}{L}-1\right) \gamma_{j} \phi_{k i 0}^{\prime \prime}\left(e_{j}\right)
$$


i.e.

$$
C_{k j}=\sum_{j=1}^{N} \frac{e_{j}}{L} \gamma_{j} \phi_{k i 0}^{\prime \prime}\left(e_{j}\right)+\sum_{i=j+1}^{N} \gamma_{i} \phi_{k 0}^{\prime \prime}\left(e_{i}\right) ; D_{k j}=-\sum_{i=1}^{j} e_{i} \gamma_{i} \phi_{k 0}^{\prime \prime}\left(e_{i}\right), j=1, \ldots, N .
$$

Substituting (28) into (26) and taking account of (27) yield

$$
\omega_{k}^{2}=\frac{\omega_{k 0}^{2}\left[1+2 \sum_{j=1}^{N} \bar{\gamma}_{j} \sin ^{2} k \pi \bar{e}_{j}\right]}{\left[1+4 \sum_{j=1}^{N} \bar{\gamma}_{j} \sin ^{2} k \pi \bar{e}_{j}+\frac{2(k \pi)^{4}}{3} \sum_{i, j=1}^{N} q_{i j} \bar{\gamma}_{i} \sin k \pi \bar{e}_{i} \cdot \bar{\gamma}_{j} \sin k \pi \bar{e}_{j}\right]},
$$

where $\omega_{k 0}=(k \pi / L)^{2} \sqrt{E I / \rho F}$ and

$$
\begin{gathered}
\bar{\gamma}_{j}=\gamma_{j} / L ; \quad \bar{e}_{j}=e_{j} / L ; \quad q_{i j}=0, i \succ j ; \\
q_{j j}=\bar{e}_{j}^{2}\left(1-\bar{e}_{j}\right)^{2} ; \quad q_{i j}=\bar{e}_{i}\left(1-\bar{e}_{j}\right)\left[1-e_{i}^{2}-\left(1-\bar{e}_{j}\right)^{2}\right], i \prec j .
\end{gathered}
$$

If the crack extents are small, i.e. $\gamma_{j}=\varepsilon \eta_{j}$, in the first order with respect to the small parameter $\varepsilon$, the equation (29) is simplified to

$$
\omega_{k}^{2}=\omega_{k 0}^{2}\left[1-2 \sum_{j=1}^{N} \bar{\gamma}_{j} \sin ^{2} k \pi \bar{e}_{j}\right] .
$$

In a particularity, for a beam with single crack, equations (29), (30) become

$$
\begin{gathered}
\omega_{k}^{2}=\frac{\omega_{k 0}^{2}\left[1+2 \bar{\gamma} \sin ^{2} k \pi \bar{e}\right]}{1+4 \bar{\gamma} \sin ^{2} k \pi \bar{x}_{c}+2 \bar{\gamma}^{2}\left[\left(\bar{e}^{2}(1-\bar{e})^{2}\right) / 3\right](k \pi)^{4} \sin ^{2} k \pi \bar{e}}, \\
\hat{\omega}_{k}^{2}=\omega_{k 0}^{2}\left[1-2 \bar{\gamma} \sin ^{2} k \pi \bar{e}\right] .
\end{gathered}
$$

Formulas similar to the equation (32) have been given in $[2,3,4]$ and a particular case of the equation (31) when $k=1$, i. e. for the fundamental frequency, was obtained by J. Fernandez-Saez, L Rubio and C. Navarro [5].

\subsection{Cantilevered beam}

For a cantilever beam, choosing $C_{k 0}=D_{k 0}=0$, i. e.

$$
C_{k j}=\sum_{i=1}^{j} \gamma_{i} \phi_{k i 0}^{\prime \prime}\left(e_{i}\right) ; \quad D_{k j}=-\sum_{i=1}^{j} e_{i} \gamma_{i} \phi_{k i 0}^{\prime \prime}\left(e_{i}\right), j=1, \ldots, N .
$$

and mode shape

$$
\begin{gathered}
\phi_{k 0}(x)=A_{k}\left[\cosh \left(\lambda_{k 0} x / L\right)-\cos \left(\lambda_{k 0} x / L\right)\right]-\left[\sinh \left(\lambda_{k 0} x / L\right)-\sin \left(\lambda_{k 0} x / L\right)\right] \\
\omega_{k 0}^{2}=\lambda_{k 0}^{4} E I / \rho F L^{4} ; A_{k}=\left[\sinh \lambda_{k 0}+\sin \lambda_{k 0}\right] /\left[\cos \lambda_{k 0}+\cosh \lambda_{k 0}\right] \\
\lambda_{10}=1.8751, \lambda_{20}=4.6941, \lambda_{30}=7.8747, \lambda_{40}=10.9955, \lambda_{50}=14.1372, \ldots
\end{gathered}
$$


satisfying the boundary conditions $\phi_{k}(0)=\phi_{k}^{\prime}(0)=\phi_{k}^{\prime \prime \prime}(L)=\phi_{k}^{\prime \prime}(L)=0$, results in the equations

$$
\omega_{k}^{2}=\frac{\omega_{k 0}^{2}\left[1+\sum_{j=1}^{N} \bar{\gamma}_{j} \bar{\Phi}_{k}^{2}\left(\bar{e}_{j}\right)\right]}{1+2 \sum_{j=1}^{N} \bar{\gamma}_{j} \bar{\Phi}_{k}^{2}\left(\bar{e}_{j}\right)+\left(\lambda_{k 0}^{4} / 3\right)\left[\sum_{i, j=1}^{N} q_{j i} \bar{\gamma}_{j} \bar{\gamma}_{i} \bar{\Phi}_{k}\left(\bar{e}_{j}\right) \bar{\Phi}_{k}\left(\bar{e}_{i}\right)\right]}
$$

with the notations $\omega_{k 0}=\lambda_{k 0}^{2} \sqrt{E I / \rho F}$ and

$$
\begin{gathered}
\bar{\Phi}_{k}(x)=\left[\cosh \left(\lambda_{k 0} x\right)+\cos \left(\lambda_{k 0} x\right)\right]-A_{k}^{-1}\left[\sinh \left(\lambda_{k 0} x\right)+\sin \left(\lambda_{k 0} x\right)\right] ; \\
\bar{\gamma}_{j}=\gamma_{j} / L, \bar{e}_{j}=e_{j} / L ; q_{i j}=0, i \succ j, \\
q_{j j}=\left(1-\bar{e}_{j}\right)^{3}, q_{i j}=\left(1-\bar{e}_{j}\right)\left[\left(1-\bar{e}_{i}\right)^{2}+\left(1-\bar{e}_{i}\right)\left(1-\bar{e}_{j}\right)-\left(\bar{e}_{j}-\bar{e}_{i}\right)^{2}\right], i \prec j .
\end{gathered}
$$

The first order approximation with respect to small crack depth of the equation (35) is

$$
\omega_{k}^{2}=\omega_{k 0}^{2}\left[1-\sum_{j=1}^{N} \bar{\gamma}_{j} \bar{\Phi}_{k}^{2}\left(e_{j}\right)\right] .
$$

For a cantilever beam with a single crack, equations (35) and (37) are reduced to

$$
\bar{\omega}_{k}=\frac{\omega_{k}}{\omega_{k 0}}=\left[\frac{\left[1+\bar{\gamma} \bar{\Phi}_{k}^{2}(\bar{e})\right]}{1+2 \bar{\gamma}\left[1+\lambda_{k 0}^{4} \bar{\gamma}(1-\bar{e})^{3} / 6\right] \bar{\Phi}_{k}^{2}(\bar{e})}\right]^{1 / 2}
$$

and

$$
\tilde{\omega}_{k}=\left[1-\bar{\gamma} \bar{\Phi}_{k}^{2}(\bar{e})\right]^{1 / 2} .
$$

In the case of two cracks, corresponding equations are

$$
\bar{\omega}_{k}=\left[\frac{\left[1+\bar{\gamma}_{1} \bar{\Phi}_{k}^{2}\left(\bar{e}_{1}\right)+\bar{\gamma}_{2} \bar{\Phi}_{k}^{2}\left(\bar{e}_{2}\right)\right]}{1+2\left[\bar{\gamma}_{1} \bar{\Phi}_{k}^{2}\left(\bar{e}_{1}\right)+\bar{\gamma}_{2} \bar{\Phi}_{k}^{2}\left(\bar{e}_{2}\right)\right]+\Psi\left(\bar{\gamma}_{1}, \bar{\gamma}_{2}, \bar{e}_{1}, \bar{e}_{2}\right)}\right]^{1 / 2},
$$

where $\Psi=\frac{\lambda_{k 0}^{4}}{3}\left[\bar{\gamma}_{1}^{2}\left(1-\bar{e}_{1}\right)^{3} \bar{\Phi}_{k}^{2}\left(\bar{e}_{1}\right)+\bar{\gamma}_{2}^{2}\left(1-\bar{e}_{2}\right)^{3} \bar{\Phi}_{k}^{2}\left(\bar{e}_{2}\right)+\bar{\gamma}_{1} \bar{\gamma}_{2} g\left(\bar{e}_{1}, \bar{e}_{2}\right) \bar{\Phi}_{k}\left(\bar{e}_{1}\right) \bar{\Phi}_{k}\left(\bar{e}_{2}\right)\right]$ with $g\left(\bar{e}_{1}, \bar{e}_{2}\right)=\left(1-\bar{e}_{2}\right)\left[\left(1-\bar{e}_{1}\right)^{2}+\left(1-\bar{e}_{1}\right)\left(1-\bar{e}_{2}\right)-\left(\bar{e}_{2}-\bar{e}_{1}\right)^{2}\right]$ and

$$
\tilde{\omega}_{k}=\left[1-\bar{\gamma}_{1} \bar{\Phi}_{k}^{2}\left(\bar{e}_{1}\right)-\bar{\gamma}_{2} \bar{\Phi}_{k}^{2}\left(\bar{e}_{2}\right)\right]^{1 / 2} .
$$

Thus, an explicit expression of natural frequencies through crack positions and extents for multiple cracked beam has been obtained in a form convenient for frequency analysis as well as multi-crack detection of beam. To validate applicability of the obtained above analytical formulas to practice, numerical calculation will be carried out below and results will be compared with those obtained by using the characteristic equation and experimental ones. 


\section{NUMERICAL INVESTIGATION AND COMPARISON}

Numerical investigation carried out in this section for illustration and validating the theory is devoted to a cantilevered beam with a single and double cracks. In all the cases, the crack extent is calculated as function of crack depth $a$ by the formulae

$$
\bar{\gamma}=\frac{E I}{L K}=(5.346 h / L) I(\delta),
$$

with $\delta=a / h$ ( $h$ is beam thickness) and

$$
\begin{aligned}
I(\delta) & =1.8624 \delta^{2}-3.95 \delta^{3}+16.375 \delta^{4}-37.226 \delta^{5}+76.81 \delta^{6}- \\
& -126.9 \delta^{7}+172 \delta^{8}-143.97 \delta^{9}+66.56 \delta^{10} .
\end{aligned}
$$

Beam with single crack

The first frequency ratio calculated by using equation (39) and those obtained from the characteristic equation $[6,7,8]$ as functions of crack position with different relative crack depth $\delta=0.1-0.6$ (i. e. crack depth of 10-60\% beam thickness) are shown in Fig. 1. Graphics in the figure show that the equation (39) is almost equivalent to the characteristic equation in calculating the fundamental frequency of cracked beam. For higher frequencies the equation (40) gives better results than the equation (39) in comparison with solution of the characteristic equation.

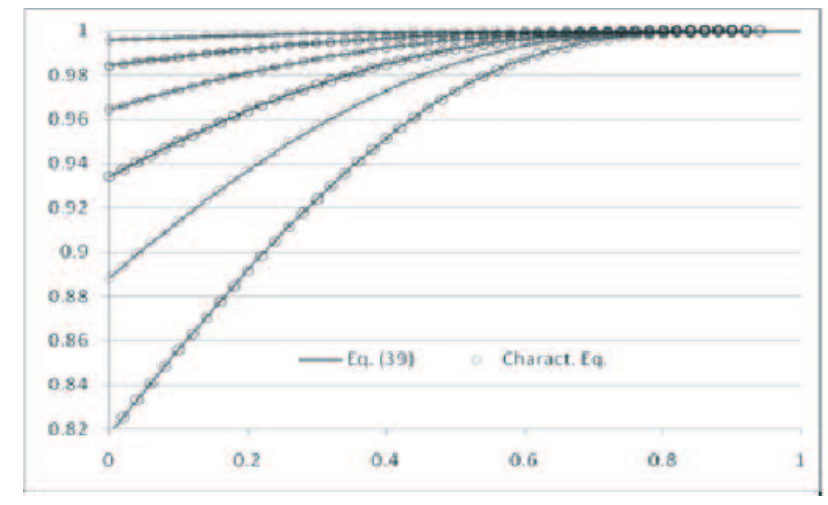

Fig. 1. Comparison of first frequency ratio computed by equation (39) with that obtained from the characteristic Equation for crack depth ratios from 0.1 to 0.6

Fig. 2 shows the second frequency ratio computed from equation (40) and characteristic equation for relative crack depth running from 0.1 to 0.4 . It can be seen from the figure that difference between frequencies calculated from equation (40) and characteristic equation has been visible only when crack depth reaches $40 \%$ beam thickness.

Beam with double cracks

First, three natural frequencies ratios (cracked to uncracked) computed from equations (41) and (42) are compared with the experimental results obtained in [12] for a cantilever with two cracks $\bar{e}_{1}=0.3175, \gamma_{1}=0.2, \bar{e}_{2}=0.6812, \gamma_{2}=0.3$ and shown in Table 1. From the table it can be seen that equation (41) results in frequencies really more close to the experimental ones than the equation (42) and error of both the equations for all 


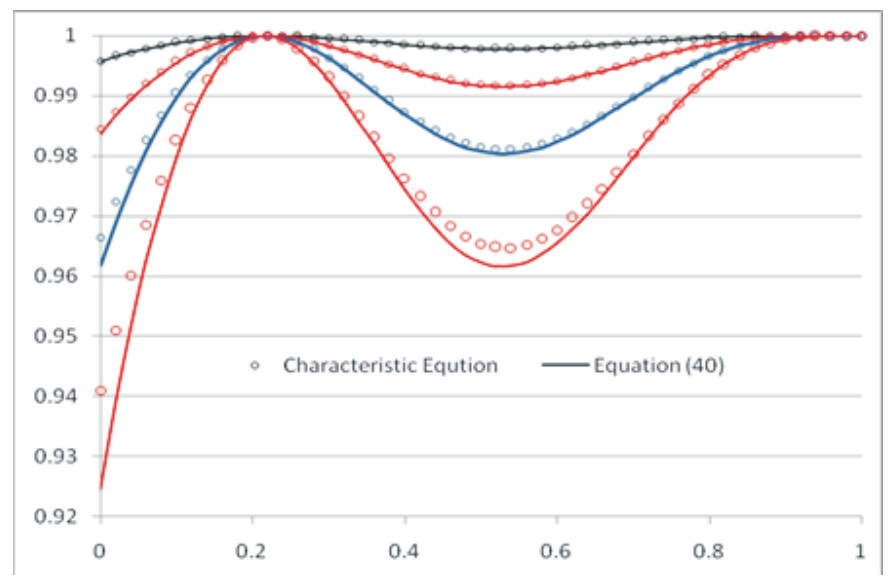

Fig. 2. Comparison of second frequency ratio computed by Eq. (40) with solution of Characteristics Eq. for crack depth ratios from 0.1 to 0.4

three frequencies does not exceed $1 \%$. This fact confirms validity of the formulas derived above.

Table 1. Comparison of three frequencies ratios with experimental results [11]

\begin{tabular}{|c|c|c|c|c|c|}
\hline Frequencies & Eq. (42) & Eq. (41) & Exp. [12] & Err. of (41)(\%) & Err. of (42)(\%) \\
\hline First & 0.993866 & 0.993938 & $\mathbf{0 . 9 9 4 5 8 1}$ & 0.064637 & 0.07188 \\
\hline Second & 0.98574 & 0.982846 & $\mathbf{0 . 9 8 1 3 6 1}$ & -0.15133 & -0.44621 \\
\hline Third & 0.971835 & 0.9696 & $\mathbf{0 . 9 6 4 2 6 5}$ & -0.5533 & -0.78511 \\
\hline
\end{tabular}

After comparing frequencies computed by FEM with the experimental results obtained by the authors of J. Lee [11] has suggested in [12] a correction of the formulae (43) to get numerical results more close to the measured one. Of course, crack model has a considerable effect on numerical analysis of cracked structures, but disagreement between predicted and measured data can arose also from the inaccurate model parameters such as material constants $(E, \rho, \nu)$ or geometrical one $(A, I, b, h)$ and from the non-ideal boundary conditions in experiments. Therefore, only crack model correction is insufficient to agree prediction with experiments. In this study, numerical results are in good agreements with experiments with no crack model correction because they have been obtained in non-dimensional form that are free from inaccuracy of the model parameters.

\section{CONCLUSION}

The main result of this study is the Rayleigh's quotient developed for multiple cracked beam in an analytical expression relating directly the frequencies to crack parameters, which can be used to calculate natural frequencies of all modes with different cases of boundary conditions. The improvement has been made based on the special choosing the mode shape of cracked beam consisting of mode shape of uncracked beam and linear functions satisfying the continuity conditions at cracks. In the case of multiple cracked 
beam with simple supports, general formulae has been derived for calculating natural frequencies that contains as a particularity the formulae obtained by J. Fernandez-Saez, L Rubio and C. Navarro [5] for fundamental frequency. Asymptotic approximations of the obtained herein formulas are similar to those given by former authors as R.Y. Liang and his coworkers [2], A. Morassi [3] and Y. Narkis [4]. The corresponding formulas have been obtained also for a cantilever beam and are applied to numerical investigation of natural frequencies in comparison with solutions of the characteristic equation given in $[6,7,8]$. Further study should be carried out in developing formulas for a nonuniform beam with different boundary conditions and using the direct relationship between frequencies and crack parameters to multi-crack detection for beam.

\section{ACKNOWLEDGEMENT}

The authors have a great pleasure to thank the NAFOSTED of Vietnam for financial support in completing this paper.

\section{REFERENCES}

[1] R. Y. Liang, J. Hu and F. Choy, Theoretical Study of Crack-Induced Eigenfrequency Changes on Beam Structures, Journal of Engineering Mechanics, 118(2) (1992) 384-396.

[2] R. Y. Liang, J. Hu and F. Choy, Quantitative NDE Technique for Assessing Damages in Beam Structures, Journal of Engineering Mechanics, 118(7) (1992), 1468-1487.

[3] A. Morassi, Crack-Induced Changes in Eigenparameters of Beam Structures, Journal of Engineering Mechanics, 119(9) (1993), 1798-1803.

[4] Y. Narkis, Identification of crack location in vibrating simply supported beams, Journal of Sound and Vibration, 172 (1994), 549-558.

[5] J. Fernandez-Saez, L Rubio and C. Navarro, Approximate calculation of the fundamental frequency for bending vibrations of cracked beam, Journal of Sound and Vibration, 225(2) (1999), 345-352.

[6] N.T. Khiem and T.V. Lien, A simplified method for frequency analysis of multiple cracked beam, Journal of Sound and Vibrations, 245(1) (2001), 737-751.

[7] D.P. Patil and S.K. Maiti, Detection of multiple cracks using frequency measurements, Engineering Fracture Mechanics, 70(12) (2003), 1553-1572.

[8] S. Caddemi and I. Caliò, Exact closed-form solution for the vibration modes of the EulerBernoulli beam with multiple open cracks, Journal of Sound and Vibration, 327(3-5) (2009), 473-489.

[9] J. Lee, Identification of multiple cracks in a beam using natural frequencies, Journal of Sound and Vibration, 320 (2009), 482-490.

[10] D.Y. Zheng and S.C. Fan, Natural frequencies of a non-uniform beam with multiple cracks via modified Fourier series, Journal of Sound and Vibration, 242(4) (2001), 701-717.

[11] R. Ruotolo and C. Surace, Damage assessing of multiple cracked beam: numerical results and experimental validation, Journal of Sound and Vibration, 206(4) (1997), 567-588.

[12] J. Lee, Identification of multiple cracks in a beam using vibration amplitudes, Journal of Sound and Vibration, 326 (2009), 205-212. 